\title{
GUIDO MÜNCH: AN APPRECIATION
}

Donald E. Osterbrock 


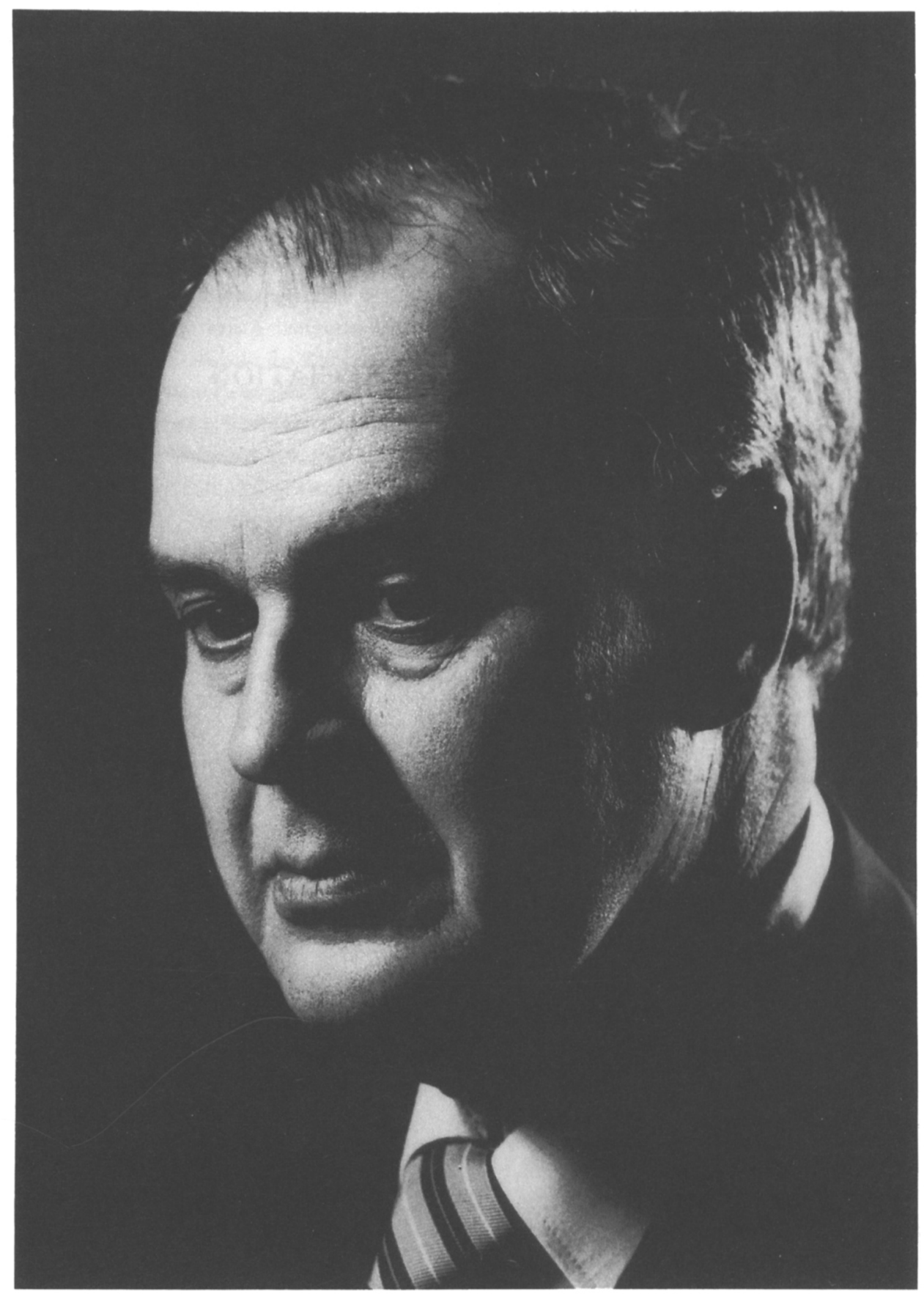

Professor GUIDO MÜNCH 
Guido Münch was born in the state of Chiapas, Mexico on June 9, 1921. He received his undergraduate education at the Universidad Nacional Autonoma de Mexico, where he began in civil engineering, but then switched to mathematics. He earned his B. S. in 1938. Two of his early heroes were mathematicians George D. Birkhoff and Solomon Lefschetz, who visited Mexico in his student days. Guido continued at UNAM as a graduate student, and two of his teachers who influenced him greatly were Spanish refugees who had fied to Mexico after the fall of the Republic, Pedro Carrasco, the former director of the Madrid Observatory, who taught astronomy, and Blas Cabrera, who taught atomic physics. Guido was also inspired by the group of American astronomers, including Walter S. Adams, Henry Norris Russell, Joel Stebbins, Harlow Shapley, Otto Struve, Donald H. Menzel, Cecilia Payne-Gaposchkin, W. W. Morgan, and Nicholas U. Mayall, who came to Mexico in early 1942 to participate in the inauguration of the Tonantzintla Observatory. As a result of this meeting Struve later offered Guido a position as a night assistant at McDonald Observatory. He accepted and after one month of training at Yerkes Observatory (April 1943), went to McDonald where he worked until the fall. Then he returned to Yerkes as a graduate student of the University of Chicago, of which the observatory is part.

Guido was a graduate student at Yerkes for three years, and earned his Ph.D. in 1946. He worked on spectroscopy of stars with Struve and Morgan, but chiefly on radiative transfer and the theory of stellar atmospheres with S. Chandrasekhar, his thesis adviser, applying Chandra's new theoretical methods to the Sun and stars, and extending them as well.

After receiving his Ph.D. Guido returned to a faculty appointment at UNAM, but came back to Yerkes as an instructor in the fall of 1947 . There he continued very creatively his stellar atmospheres research. With Chandra he also undertook a theoretical discussion of the statistics of stellar rotation velocities, and their joint paper on this subject was an important one. Guido was promoted to assistant professor in 1949. Yerkes Observatory was a center of research on interstellar matter, and Guido became interested in the cloud 
structure of interstellar matter as revealed by the fluctuations in brightness in the Milky Way. He and Chandra developed the theory of these fluctuations, and applied them to deriving parameters of the cloud structure, in an important series of papers.

Guido went to the California Institute of Technology in 1951 as an assistant professor and staff member of the Mount Wilson and Palomar Observatories. In 1948 Jesse L. Greenstein had been the first to move there from Yerkes, to start the Caltech Astrophysics Department in its modern form; he brought Guido to join him three years later, myself two years after that, and Arthur D. Code three years still later, all of us Yerkes Ph.D.'s. At Caltech Guido was promoted to associate professor in 1954, and to full professor in 1959. He became a U.S. citizen in 1957.

In research he continued his work on stellar atmospheres, especially on hot stars, and most particularly on hot population II "subdwarfs" (O stars). With the coude spectrograph of the 200-inch Hale telescope Guido opened a new era in the study of interstellar absorption lines, going beyond the outstanding work Adams had done with the 100-inch. Guido, with the telescope necessary to observe the entire Milky Way, obtained high-dispersion spectrograms which not only clearly resolved the absorption lines into "cloud" components, but which also showed groups of them in the outer Perseus spiral arm, well separated from the clouds in our (Orion) arm. It was widely recognized as a very important direct confirmation of the nature and location of the spiral arms in our Galaxy. Guido's papers and review articles on the interstellar medium are classics. He also used $\mathrm{O}$ stars in high galactic latitudes to study interstellar gas far from the plane. Everyone knew "it should not be there," but Guido showed that it is, and that it is infalling.

He also carried out very high-dispersion emission-line studies, especially of the Orion Nebula. He began this work with Olin C. Wilson, who had been applying these methods to planetary nebulae. Guido was always very interested in instruments, especially in pushing the limits of wavelength resolution. Beginning with this work on Orion, he became more and more active in conceiving new methods, improvements in existing instruments, finally whole new instruments, working closely with students, postdocs, engineers and technicians to bring them to reality and practice. He made especially important studies of the velocity and density fields within the Orion nebula, and of the neutral gas in it.

Guido also produced many new results on the physics of planets. He was one of the first well known U. S. observational astronomers to take the space program seriously and to get actively involved in it himself. With Hyron Spinrad and Lewis D. Kaplan he made the first detection of $\mathrm{H}_{2} \mathrm{O}$ vapor on Mars, by high-dispersion near-infrared spectroscopy. Their spectroscopic measurement of the density of Mars's atmosphere (from the column density of $\mathrm{CO}_{2}$ they determined from their spectra) was crucial in planning the first Martian landers. Guido also pioneered in space astrophysics in applying infrared 
measurements, especially to Jupiter and its atmosphere, Mars, and Venus (the Mariner missions). He worked with Gerry Neugebauer, Lawrence Trafton, and many other former Caltech graduate students on these subjects.

With the 200-inch Hale telescope Guido also worked on interstellar matter in and near the nucleus of M 31, and with his brother Luis on the rotational velocities in the inner part of our Galaxy (from stellar spectra). Guido's pioneering theoretical paper on L $\alpha$ in interstellar matter, and how it is resonance-scattered by $\mathrm{H}$ atoms and destroyed by absorption by dust, is an extremely important one. He has always tried to use instruments to their limits, and beyond. He has thought creatively of problems that needed to be solved, of how they could be solved with high spectral resolution, and then he has gone out and solved them. He had the theoretical insights, the mathematical facility, and the instrumental skills to do so.

In 1977 Guido left Caltech to accept the position of co-director of the Max-PlanckInstitut für Astronomie in Heidelberg. There, with Hans Elsässer, he created a school of high-resolution spectroscopy. The work of this whole group is well known from their many important papers in Astronomy and Astrophysics.

Guido has always kept close connections with his native land and with Mexican astronomy. He returned to Mexico often, and advised many Mexican students informally about astronomical opportunities in the United States.

The astrophysics graduate students who did their Ph.D. theses under Guido's supervision at Caltech form an imposing list: the late John C. Stewart, Manuel Mendez, Robert Norton, Robert A. R. Parker, Lawrence Trafton, James G. Gunn, Richard Larson, Christopher M. Anderson, Jeffrey Scargle, Bruce A. Peterson, Vicky A. Peterson, Virginia Trimble, Jay Frogel, S. Eric Persson, Judith Cohen and Jorge Melnick. He also had Robert Brinkman as a Ph.D. thesis student in aeronomy. In addition, he has had two more doctoral thesis students at Heidelberg, P. Gomez-Garrido and Roland Gredel.

Guido was elected to the American Academy of Arts and Sciences in 1962, and to the U. S. National Academy of Sciences in 1967. He was awarded the NASA Medal for Exceptional Scientific Achievement in 1974, and Spain's Prince of Asturias Prize for Scientific and Technical Research in 1989. He was elected to the Third World Academy of Sciences in 1984. He is an outstanding research scientist, and I am proud to have been his student, colleague, friend and admirer for forty years.

I am very grateful to S. Chandrasekhar, J. L. Greenstein, A. D. Code and E. E. Mendoza for their memories and thoughts, which I have incorporated with my own in this appreciation. 